\title{
Small PARP inhibitor PJ-34 induces cell cycle arrest and apoptosis of adult T-cell leukemia cells
}

\author{
Xue Tao Bai ${ }^{\dagger}$, Ramona Moles ${ }^{\dagger}$, Hassiba Chaib-Mezrag and Christophe Nicot ${ }^{*}$
}

\begin{abstract}
Background: HTLV-I is associated with the development of an aggressive form of lymphocytic leukemia known as adult T-cell leukemia/lymphoma (ATLL). A major obstacle for effective treatment of ATLL resides in the genetic diversity of tumor cells and their ability to acquire resistance to chemotherapy regimens. As a result, most patients relapse and current therapeutic approaches still have limited long-term survival benefits. Hence, the development of novel approaches is greatly needed.

Methods: In this study, we found that a small molecule inhibitor of poly (ADP-ribose) polymerase (PARP), PJ-34, is very effective in activating S/G2M cell cycle checkpoints, resulting in permanent cell cycle arrest and reactivation of p53 transcription functions and caspase-3-dependent apoptosis of HTLV-I-transformed and patient-derived ATLL tumor cells. We also found that HTLV-I-transformed MT-2 cells are resistant to PJ-34 therapy associated with reduced cleaved caspase-3 activation and increased expression of RelA/p65.
\end{abstract}

Conclusion: Since PJ-34 has been tested in clinical trials for the treatment of solid tumors, our results suggest that some ATLL patients may be good candidates to benefit from PJ-34 therapy.

\section{Introduction}

Human T-cell leukemia virus type I (HTLV-I) is etiologically linked to the development of an aggressive type of peripheral T-cell leukemia known as ATLL [1]. The clinical course varies among infected patients and the disease has been classified into four distinct entities: smoldering, chronic, acute, or lymphoma [2]. Although many features of HTLV-I biology have been discovered [3], the treatment of the disease remains unsatisfactory, with minimal improvements in the overall survival of patients [4]. Overall, the current therapies used for the treatment of ATLL patients in the acute phase have limited impact and the overall projected 4-year survival rate of acute ATLL is around $5 \%$ [5]. The mechanism by which HTLV-I causes ATLL is still not fully understood, but a latency period of several decades before the onset of the disease suggests that long-term survival and expansion of virus-infected cells are required. Along these lines, we have previously shown that reactivation of

\footnotetext{
* Correspondence: cnicot@kumc.edu

${ }^{\dagger}$ Equal contributors

Department of Pathology and Laboratory Medicine, Center for Viral

Oncology, University of Kansas Medical Center, 3901 Rainbow Boulevard, Kansas City, KS 66160, USA
}

telomerase activity is one of the essential steps in the transformation process of HTLV-I-infected cells [6]. HTLV-I transformed CD4/CD25+ T cells in vivo and in vitro. In early stages, infected cells may rely on an autocrine/paracrine IL-2/IL-2R or IL-15/IL-15R cytokine loop for active proliferation [7]. During that stage, HTLV-I-infected cells accumulate genetic and epigenetic mutations and are prone to genomic instability. At the basis of this phenomenon is the viral oncoprotein Tax, which has been shown to inactivate tumor suppressors such as p16ink, p53, RB, and p21WAF [8], affect genome stability [9], and activate oncogenic signaling pathways such as NF-kB, Notch, and JAK/STAT [10-12]. In addition, Tax also induces DNA breaks during cellular replication and inhibits DNA repair pathways, leading to accumulation of genetic alterations [13, 14]. Eventually, an infected IL-2-independent transformed cell emerges with a selective growth advantage resulting in clonal expansion. The molecular basis for IL-2 independence is still unknown although a majority of HTLV-I-transformed cells simultaneously acquire constitutive JAK/STAT activation. The transition from IL-2 dependent to IL-2 independent is believed to mimic the disease progression from smoldering or chronic to the acute type of ATLL.

\section{Biomed Central}


Recently, we showed that Tax can induce genomic DNA double-strand breaks (DDSB) by targeting the fork of replication during cell division [13]. Since HTLV-Itransformed cells have a defective homologous recombination repair (HR) pathway [14], we hypothesized that HTLV-I-transformed and ATLL cells might be particularly sensitive to small drug inhibitors targeting DNA replication. Although poly (ADP-ribose) polymerase (PARP) is a single-strand break sensing protein, PARP inhibitors (PARPi) have been shown to be selectively effective in cells with an HR-defective pathway [15]. Numerous PARPi (PJ34, MK4827, ABT-888, AZD2281, and BSI-201) are in clinical trials for breast cancer, ovarian cancer, and prostate cancer $[16,17]$. The PARPi PJ-34 has been shown to cause cell cycle arrest in various human cancers, including myelodysplastic syndromes (MDS) and acute myeloid leukemia (AML) $[18,19]$.

In this study, we investigated the efficacy of the PARPi PJ-34 in targeting HTLV-I-transformed cells and a panel of patient-derived ATLL cell lines. Our results demonstrate that PJ-34 used as a single agent is a potent inhibitor of cellular growth in IL-2-dependent as well as IL-2independent transformed ATLL cells. We also found that another PARPi (olaparib/AZD2281) is also effective against HTLV-I-transformed cells. We further show that cells treated with PJ-34 reactivated p53 functions and accumulated in G2/M. Tumor cells died from apoptosis as shown by annexin $\mathrm{V}$ staining but this process appears to be largely p53 - independent since ATLL-derived cells not expressing p53 (MT-1 and ED) were still efficiently killed by PJ-34. We found that HTLV-I-transformed MT-2 and C91PL cell lines were resistant to PJ-34 treatment. We found that PJ-34-resistant cells expressed higher basal levels of Bax and were unable to engage the cleavage of pro-caspase-3. In addition, resistance of MT2 cells was independent from p53BP1 and PARP1 but coincides with activation of NF- $\mathrm{kB}$.

\section{Materials and methods}

\section{Cell lines and reagents}

HTLV-I-transformed cell lines (MT-4, MT-2, C8166, C91PL) and ATL-like cell lines, IL-2 independent (MT1, ATL-T, ED-40515(-), ALT-25), were maintained in RPMI-1640 media supplemented with $10 \% \mathrm{FBS}$, penicillin, and streptomycin. ATL-like cell lines, IL-2 dependent (ATL-43T, KOB, and ATL-55T), were maintained in RPMI-1640 supplemented with $10 \%$ FBS, penicillin, and streptomycin and IL-2 $(50 \mathrm{U} / \mathrm{mL})$. PARP1 inhibitor, PJ-34 (N-(5, 6-Dihydro-6-oxo-2-phenanthridinyl)-2-(dimethylamino) acetamide, ab120981), was purchased from Abcam. Stock solutions $(25 \mathrm{mM})$ were made with dimethyl sulfoxide (DMSO). In all experiments, cells were treated with different concentrations of PJ-34 $(5,10,25$, and $50 \mu \mathrm{M})$ or DMSO for 3 or 5 days, as indicated. Olaparib was used at $25 \mu \mathrm{M}$ for 3 days. Cell Proliferation Kit II (XTT) was purchased from Roche Life Science.

\section{Cell cycle and cell proliferation}

HTLV-I-transformed and ATL cells were treated with DMSO or PJ-34 for 3 days. The cells were collected and washed with $1 \times$ PBS and fixed with $75 \% \mathrm{EtOH}$ overnight at $-20{ }^{\circ} \mathrm{C}$. The next day, cells were washed with ice-cold $1 \times$ PBS, followed by treatment with RNase for $15 \mathrm{~min}$ at $37{ }^{\circ} \mathrm{C}$, stained with $100 \mu \mathrm{g} / \mathrm{mL}$ propidium iodine (PI) for $15 \mathrm{~min}$ at room temperature, and analyzed by flow cytometry. Cell proliferation was measured by the XTT assay. For the XTT assay, $100 \mu \mathrm{L}(10,000)$ cells were seeded in 96-well plates and treated with different concentrations of PJ-34 $(5,10,25$, and $50 \mu \mathrm{M})$ or DMSO for 5 days. A $50-\mu \mathrm{L}$ of XTT labeling mixture was added to each well and incubated for $6 \mathrm{~h}$. The absorbance was measured at 450 and $620 \mathrm{~nm}$ by spectrophotometry. Results were plotted as mean \pm SD from at least two independent experiments.

\section{Cell tracking assay}

CFDA-SE (Molecular Probes, Eugene, OR) was used to label cells according to the manufacturer's protocol. After labeling, $1 \times 10^{5}$ cells were analyzed by FACS assay for $0 \mathrm{~h}$ control. The rest of the labeled cells were divided into two groups: one treated with DMSO, the other one with PJ-34. Every $24 \mathrm{~h}, 1 \times 10^{5}$ cells were taken out for FACS assay until $72 \mathrm{~h}$.

\section{Apoptosis assays and mitochondrial membrane potential}

The cells were collected and washed with $1 \times$ PBS then stained with annexin V/propidium iodide using the Alexa Fluor ${ }^{\circ} 488$ Annexin V/Dead Cell Apoptosis Kit (Molecular Probes, Eugene, OR) according to the manufacturer's instructions. MT-4, C91PL, and MT-2 cells were treated with DMSO or PJ-34. Then, the cell mitochondrial membrane potential $(\Delta \Psi \mathrm{m})$ was measured using the JC-1 Assay Kit (Invitrogen) according to the manufacturer's instructions.

\section{Western blotting}

Total cell extracts were prepared with RIPA buffer (50 mM Tris $\mathrm{HCl}$ pH 8.0, $150 \mathrm{mM} \mathrm{NaCl}, 1$ \% NP-40, $0.5 \%$ sodium deoxycholate, $0.1 \%$ SDS). Samples were separated on SDS-PAGE followed by electroblotting to polyvinylidene difluoride membranes. The following antibodies were used: cyclin E (C-19), cyclin A (h432), cyclin B1 (H-20), p21 (C-19), actin (C-11) and p53 (FL393), Bcl-2 (100), Bax (N-20), Bcl-xS/L (S-18), caspase-8 (H-134), and caspase-3 (H-60) are all from Santa Cruz Biotechnology; Phospho-Histone H2A.X (Ser139) (p- 
H2AX, 2577) and Phospho-p53 (Ser15) (pS15-p53, $\mathrm{D} 4 \mathrm{~S} 1 \mathrm{H})$ are from Cell Signaling Biotechnology.

\section{Real-time RT-PCR}

Total RNA was prepared with TRIzol reagent (Invitrogen, Carlsbad, CA). After DNase I treatment, the RNA was reverse transcribed and the cDNA was used for PCR and real-time PCR. The real-time PCR assay was performed using the $\mathrm{iTaq}^{\mathrm{mi}}$ Universal $\mathrm{SYBR}^{\circ}$ Green Supermix. Real-time PCR was used to detect the expression of Bax, MDM2, GADD45 $\alpha$, XIAP, FLIP, p53BP1, BRCA1, p65/RELA, and PARP1. Real-time PCR was performed in duplicate, and data were normalized to GAPDH expression.

\section{Results}

PJ-34 induces cell cycle arrest in HTLV-I-transformed and ATLL-derived cell lines

HTLV-I-transformed and ATLL cells are characterized by excessive chromosomal instability associated with defects in HR DNA repair pathways. We, therefore, hypothesized that these cells might be particularly sensitive to PARPi. HTLV-I-transformed T-cell lines MT-2, MT-4, C8166, and patient-derived ATLL cell lines ED and
ATL-25 were treated with PJ-34 for 3 days and cell cycle was analyzed by propidium iodide staining and DNA content analyses on live gated cells by FACS. Our results revealed a marked increase of cells in the G2/M phase of cell cycle following treatment (Fig. 1a, b). These results are consistent with previous findings that the mitotic spindle checkpoint is functional in HTLV-Itransformed and Tax-expressing cells, which arrest in G2/M following treatment with NU7026, Taxol, or Nocodazole [20, 21]. We next used carboxyfluorescein succinimidyl ester (CFSE) incorporation and confirmed that HTLV-I-transformed MT-2 cells treated with PJ-34 are no longer dividing (Fig. 1c), suggesting that these cells are arrested in all the different phases of the cell cycle. Consistent with these results, we observed a significant increase in cyclin-dependent kinase inhibitor (CDK) p21WAF expression in MT-2, C91PL, and MT-4 cells after treatment with PJ-34 (Fig. 1d). Cyclin A associates with CDK2 and is involved in the initiation and completion of DNA replication during S phase [22]. Our experiments revealed a significant decrease in cyclin A expression after treatment with PJ-34. Since cyclin A is also important for centrosome replication and G2/M transition [23], these data may provide an explanation

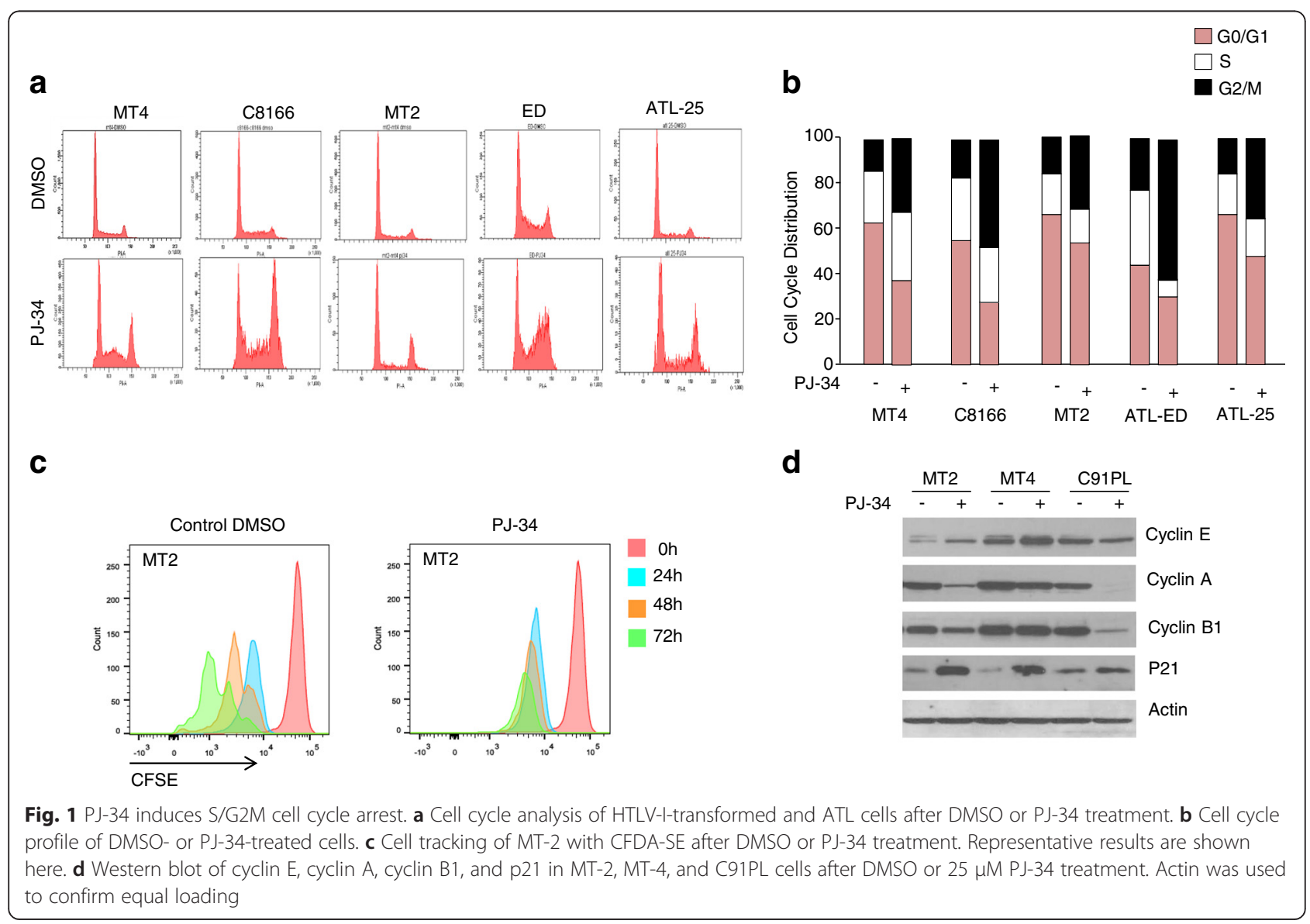


for $G 2 / M$ arrest observed in most cells after treatment with PJ-34. In contrast, cyclin E, involved in G1 exit and initiation of S phase [24], was downregulated by PJ-34 in C91PL cells only (Fig. 1d). We next investigated the expression of Cyclin $\mathrm{B} 1$ because of its role in mitosis [25]. We found that cyclin B1 expression was significantly reduced in MT-2 and C91PL but not in MT-4 transformed cells (Fig.1d). Overall, our results suggest that PARPi PJ-34 affects multiple cyclins and is effective in preventing proliferation of HTLV-I-transformed cells.

Next, we measured quantitative inhibition of cellular proliferation using XTT assays, a colorimetric assay for the quantification of cellular proliferation. These assays were performed in four HTLV-I-transformed cells and seven ATLL-derived cell lines and a dose increase of PJ-
$34(5-50 \mu \mathrm{M})$ or DMSO vehicle as a control (Fig. 2). To demonstrate specificity of PJ-34, we used human PBMCs from a HTLV-I-negative healthy donor. These studies demonstrate that IC50 for most HTLV-I-transformed or ATL cells is around $10 \mu \mathrm{M}$ (MT-1 and C91PL $20 \mu \mathrm{M}$ ) (Fig. 2). Even at $50 \mu \mathrm{M}$ of PJ-34, PBMCs present less than $25 \%$ inhibition when most HTLV-I-transformed cells had more than $80 \%$ inhibition (Fig. 2).

\section{PJ-34 induces p53-dependent and p53-independent apoptosis in HTLV-I-transformed and ATLL-derived cell lines}

PARPi have been used to induce apoptosis in breast cancer and ovarian cancer cells [26-28]. The lack of effective therapy for ATLL prompted us to investigate the cytotoxicity of PJ-34 in four HTLV-I-transformed and seven ATLL-

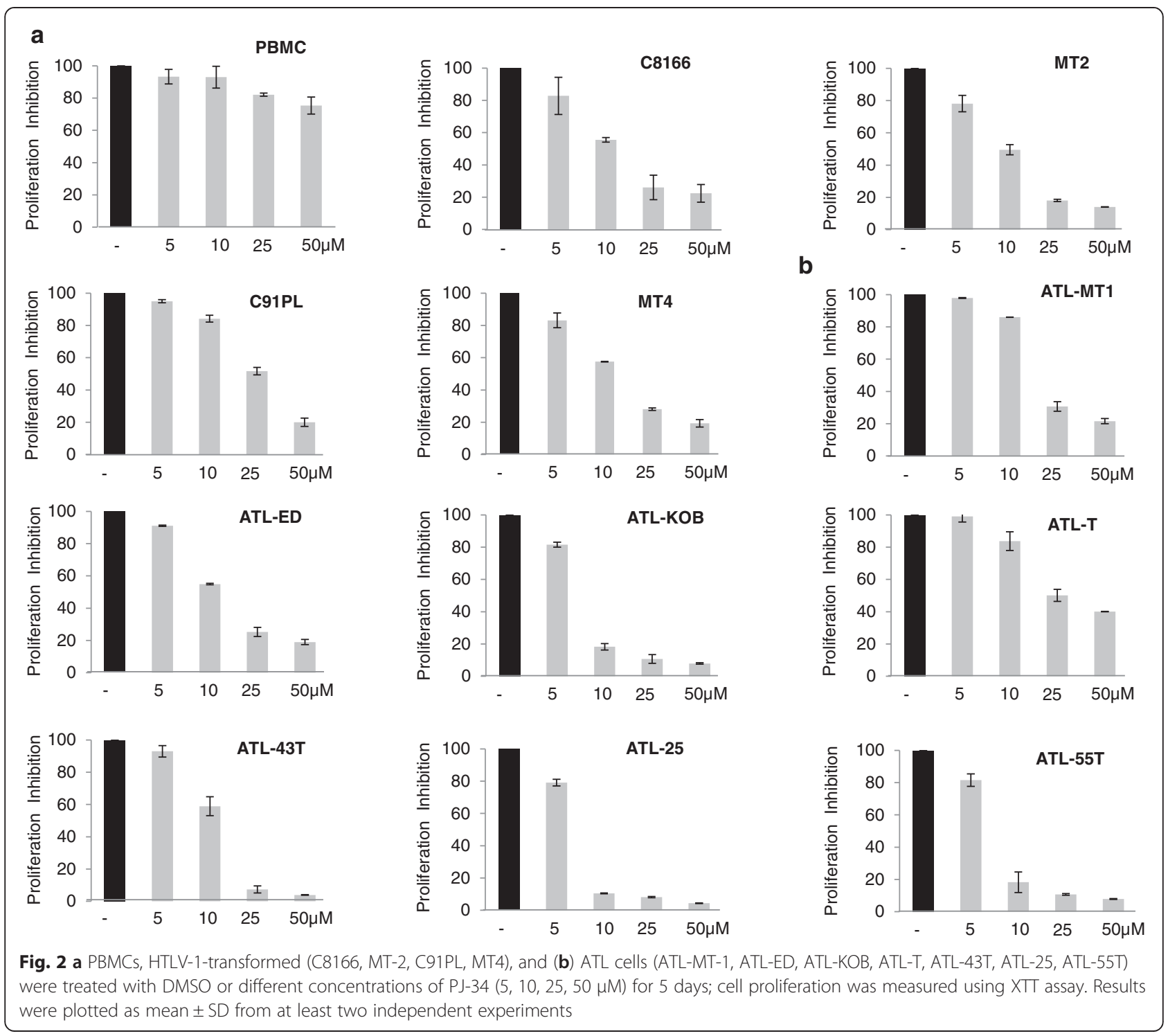


derived cell lines. Cells were treated for 5 days and analyzed by annexin V staining. Our results demonstrated a significant level of apoptosis in all patient-derived ATLL cell lines and most HTLV-I-transformed cell lines, with the exception of C91PL and MT-2 which appear to be resistant to PJ-34-induced apoptosis (Fig. 3a, b). These results were confirmed using a dose-dependent increase of PJ-34 on HTLV-I-transformed MT-4 cells (Fig. 3c). To confirm specific effects of PJ-34 on HTLV-I-transformed cells, we used a dose-dependent increase of PJ-34 and normal human PBMCs as a negative control (Fig. 4). Overall, these studies confirmed our previous observations and showed that HTLV-I-transformed cells are more sensitive than PBMC controls (Fig. 4). Also in agreement with above-mentioned data, C91PL had kinetics similar to PBMC and MT-2 was resistant to PJ-34 even at a dose of $50 \mu \mathrm{M}$ (Fig. 4). Interestingly, another PARPi, olaparib (AZD2281), also induced apoptosis in HTLV-I-transformed MT-4 and ATL-ED resulting in only 34 and $10 \%$ live cells, respectively, after 3 days of treatment. In contrast, similar to PJ-34, MT-2 cells were more resistant, with $67 \%$ live cells after 3 days of olaparib (Fig. 5).

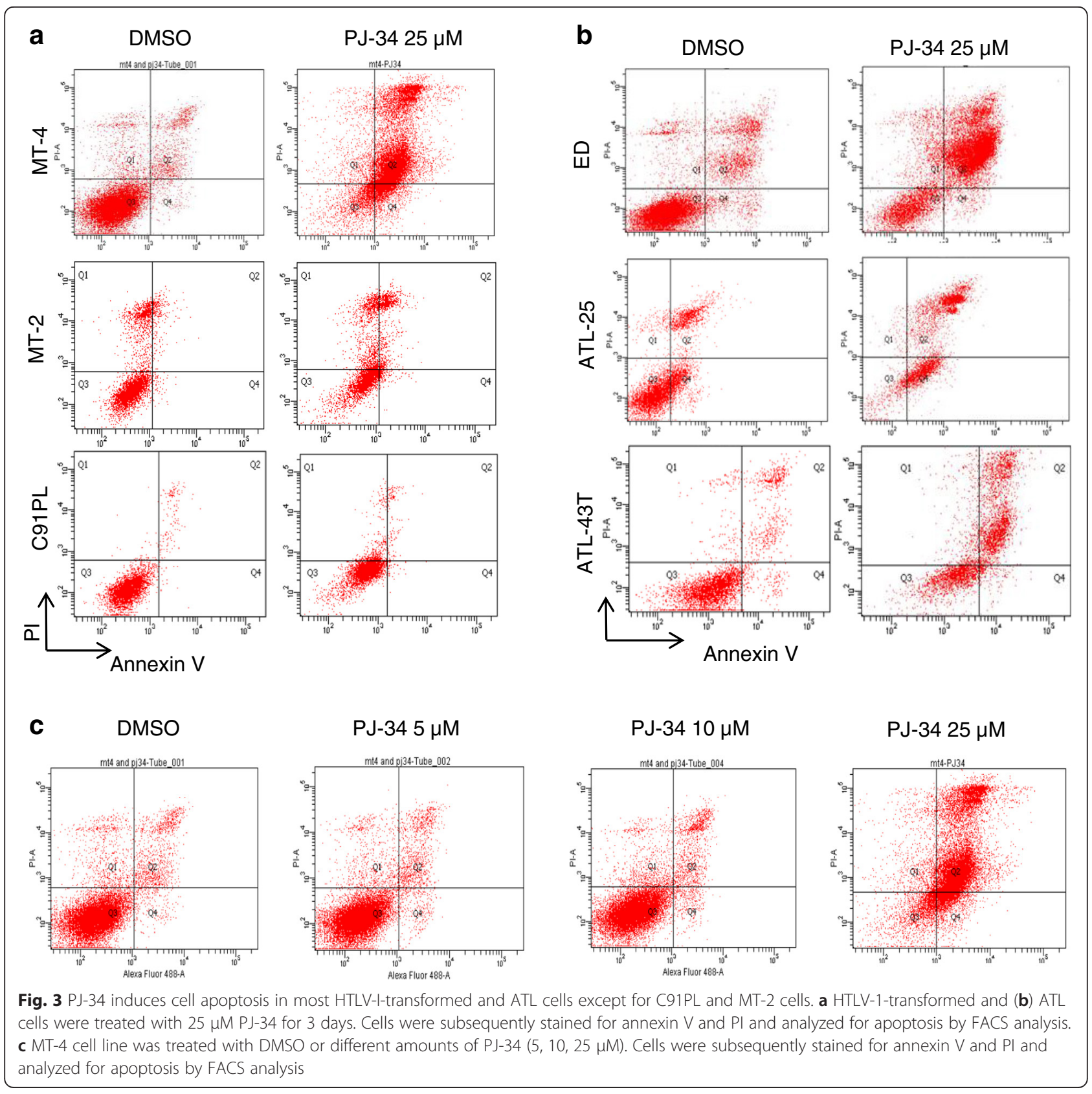




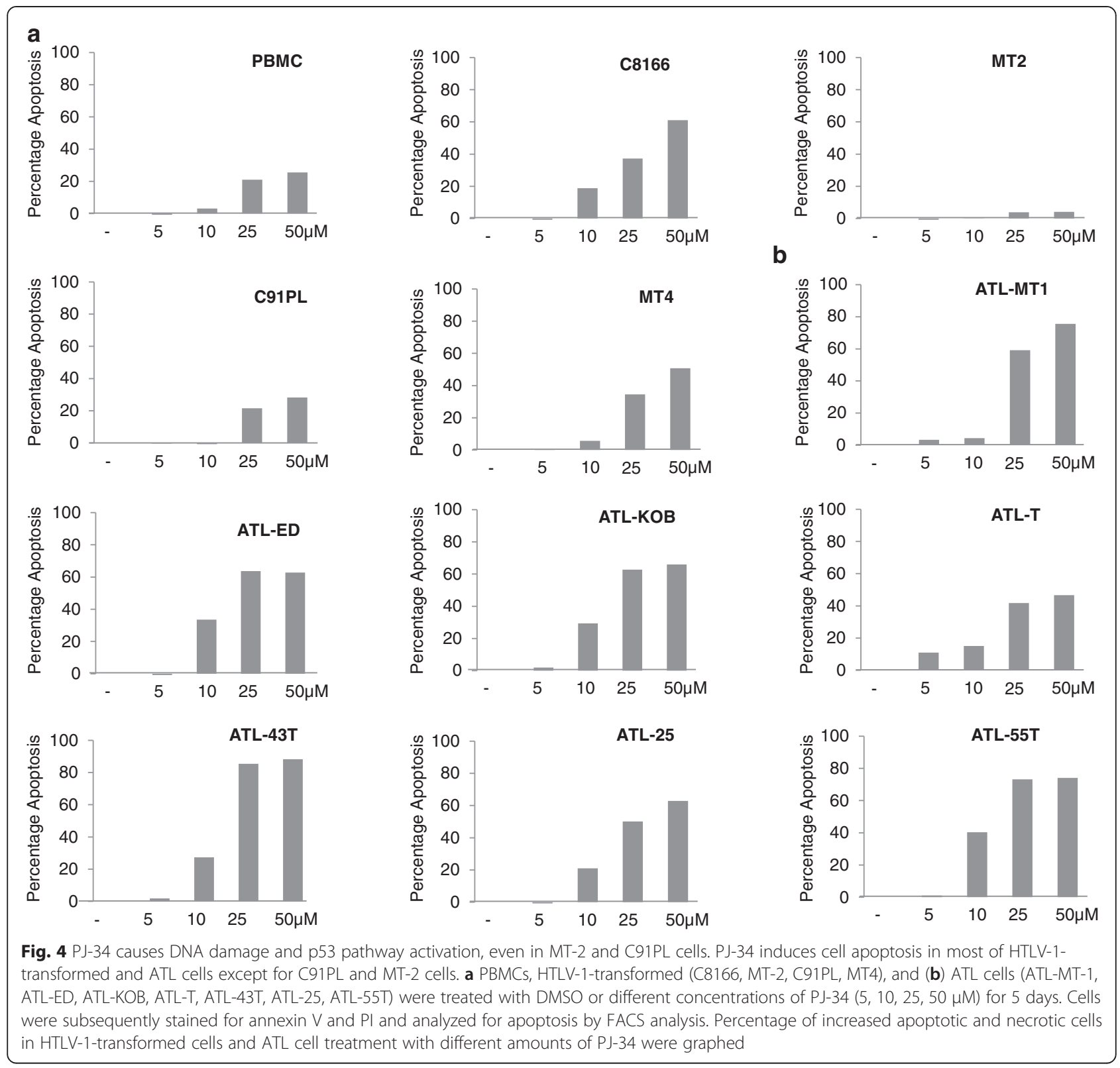

\section{PJ-34 treatment leads to reactivation of p53 functions in HTLV-I-transformed cells}

As a result of PARP inhibition by PJ-34, unrepaired single-strand DNA breaks (SSB) are converted into double-strand breaks (DDSB) at fork replication and result in accumulation of DDSB and $\mathrm{p}-\mathrm{H} 2 \mathrm{AX}$ foci. Consistent with this notion, we found an increased $\mathrm{p}$ H2AX expression in cells treated with PJ-34 (Fig. 6a). Accumulation of $\mathrm{p}-\mathrm{H} 2 \mathrm{AX}$ is known to result in ATM/ATR activation and downstream Chk1-p53 pathway. Indeed, treatment of HTLV-I-transformed cells with PJ-34 caused an increased p-p53 at serine 15 and p21WAF (Fig. 6a), suggesting that p53 may be active in these cells. Drug cytotoxicity is often associated with activation of p53-dependent apoptosis, and tumors with mutated inactive p53 are frequently resistant to radio- and chemotherapy. HTLV-Itransformed cells in vitro and in vivo have been shown to express high levels of p53 that is genetically or functionally inactivated [29-31] but can be reactivated leading to either apoptosis or senescence of HTLV-I-transformed cells [32]. We compared activation of p53-mediated transcription in MT-4 (PJ-34 sensitive) and C91PL or MT-2 (PJ-34 resistant). Our results suggested that $\mathrm{p} 53$ was functionally reactivated in all HTLV-I-transformed cells following treatment with PJ-34, as demonstrated by an increase in p53 (Fig. 6b) and increased mRNA expression of the p53 target genes 

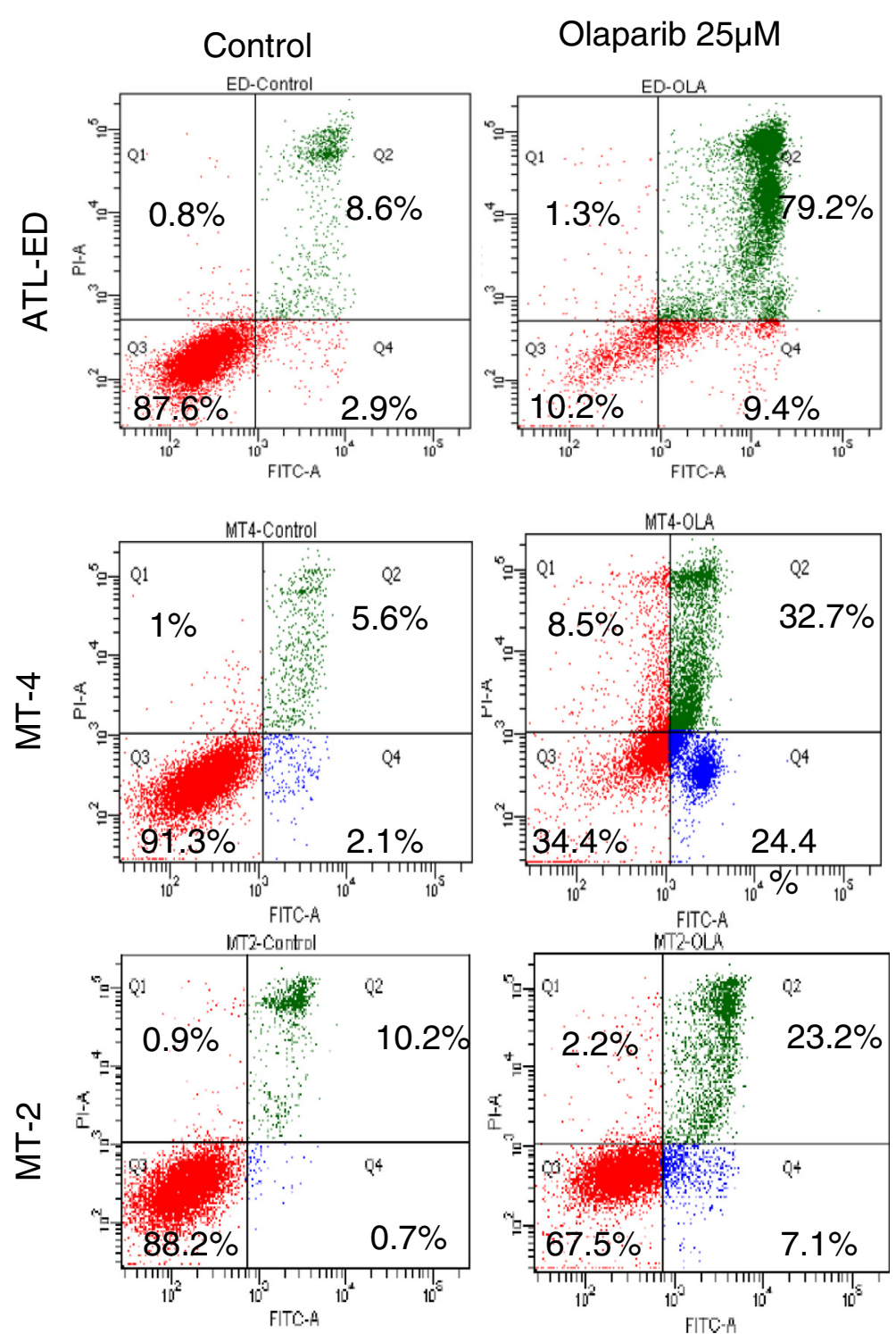

Fig. 5 PARPi olaparib (AZD2281) was used at $25 \mu \mathrm{M}$ for 3 days on HTLV-I-transformed MT-4, C91PL and MT-2 cells and apoptosis was measured by annexin $V$ staining and FACS

Bax, MDM2, p21, and GADD45 $\alpha$ (Fig. 6c-e). One common target of both p53-dependent G1 arrest and p53independent G2 arrest is p21WAF, whose expression was consistently increased after PJ-34 treatment of HTLVI-transformed cells. However, our studies suggest that the sensitivity of HTLV-I-transformed cells to PJ-34induced apoptosis may not be dependent upon p53 signaling since both MT-2 and C91PL cells were resistant to PJ-34-mediated apoptosis despite reactivation of p53 in these cells. In addition, some ATLLderived HTLV-I-transformed cells such as ED or MT1 have no detectable expression of functional p53 and yet these cells were sensitive to PJ-34 treatment (Figs. 2 and 4).

\section{Resistance of HTLV-I-transformed cells to PJ-34-induced} apoptosis is associated with defective caspase-3 activation

Disruption of the mitochondrial membrane potential, $\Delta \Psi \mathrm{m}$, represents a critical step in the activation process of apoptosis cell death. Therefore, we measured $\Delta \Psi \mathrm{m}$ in MT-4, MT-2, and C91PL cells treated with PJ-34. Our results suggested that the $\Delta \Psi \mathrm{m}$ collapse was pronounced when HTLV-I-transformed cells were treated with PJ-34, and MT-4 cells were significantly more affected than MT-2 (Fig. 7a). The collapse of the $\Delta \Psi \mathrm{m}$ is usually associated with activation of caspases. Caspase- 9 is activated immediately downstream of the mitochondria following cytochrome $\mathrm{C}$ release and apoptosome 


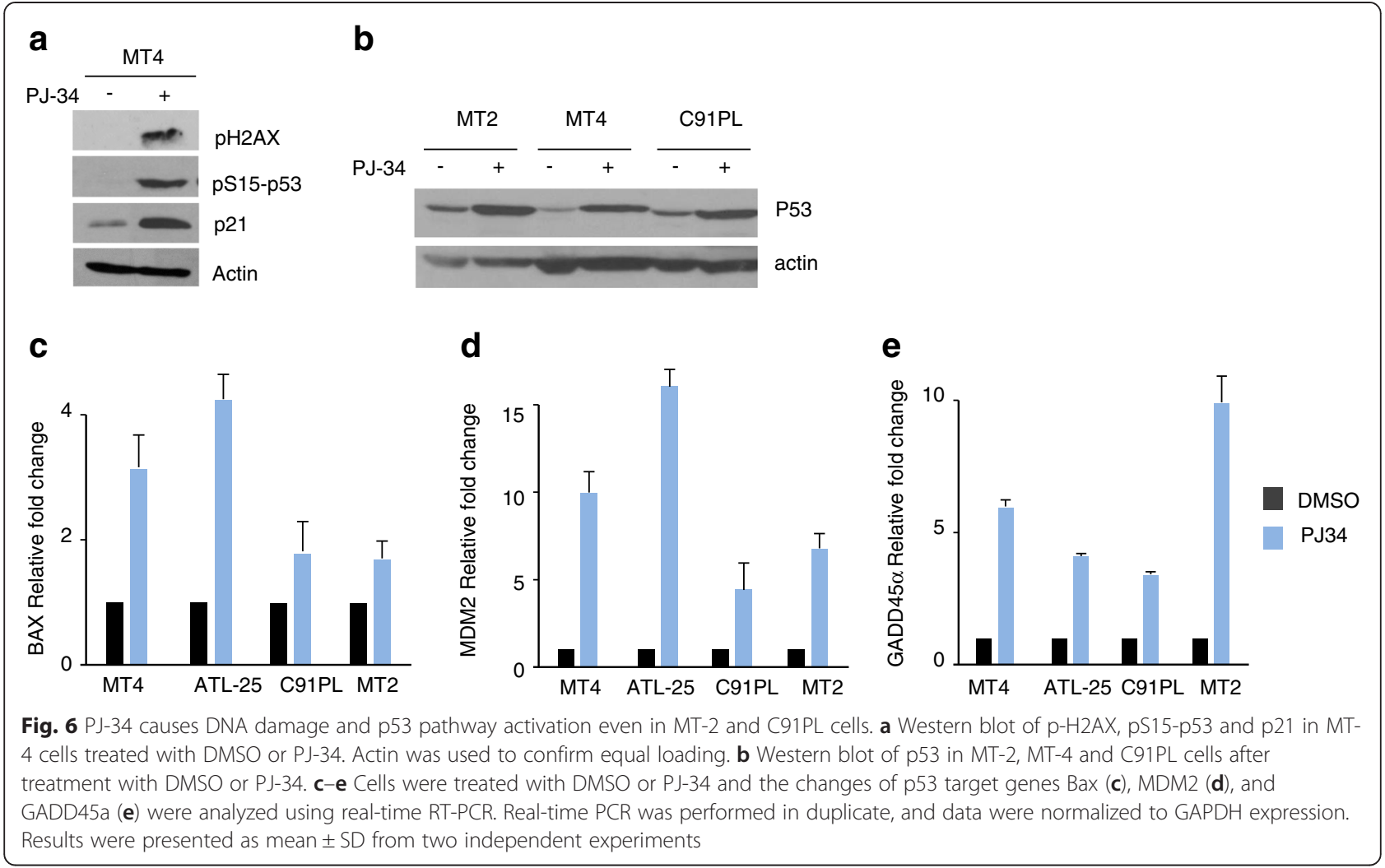

formation. In contrast, caspase- 8 is traditionally activated by death receptor signaling. These are referred to as canonical and non-canonical apoptosis pathways [33]. Caspase- 3 is a central mediator in the caspase pathway and poly (ADP-ribose) polymerase (PARP) is a substrate of caspase- 3 protease activity and its processing is usually associated with apoptotic cell death. To gain further insight into the mechanism used by MT-2 and C91PLtransformed cells to resist PJ-34 toxicity, we analyzed different markers associated with pro-apoptotic and anti-apoptotic activities. Although Bcl-xL is overexpressed and an important anti-apoptotic factor in HTLV-I cells [34], the surge in levels of Bax expression cannot be counteracted by high levels of $\mathrm{Bcl}-\mathrm{xL}$, since the latter does not prevent Bax-mediated apoptosis [35]. Expression of anti-apoptotic Bcl-2 was largely unchanged after treatment with PJ-34 (Fig. 7b). We then evaluated whether the observed apoptotic phenotype of HTLV-I-transformed cells treated with PJ-34 was dependent upon the mitochondrial pathway by assessing Bax activation and activation of caspase-3. Surprisingly, active caspase 3 cleaved products were readily detected in MT-4-treated cells but not detected in MT-2 and C91PL cells after exposure to PJ-34 (Fig. 7c). Therefore, we investigated levels of FLIP and XIAP since these are known to affect activation of caspase-3, but no difference was detected for MT-2 and C91PL cells (Fig. 7d).
We amplified and sequenced the cDNA of the caspase- 3 gene, but it was wild type in both MT-2 and C91PL. The reason for the lack of active caspase-3 in MT-2 and C91PL after PJ-34 treatment is still unclear at the moment and under further investigation.

Resistance to PJ-34 in HTLV-I-transformed MT-2 cells coincides with increased expression of RelA/p65

We next wanted to understand the molecular mechanism involved in the resistance of HTLV-I-transformed cells MT-2 and C91PL. This has direct and important implications for treatment of ATLL patients with PJ-34, and identification of resistance biomarkers could also provide new insights for PARPi treatment of breast, ovarian, and prostate cancer patients. Acquisition of resistance to PARPi can be achieved by genetic alterations that restore HR functions [36]. Frequently, increased expression of BRCA1 or $\mathrm{p} 53 \mathrm{BP} 1$ has been shown to correlate with resistance to PARPi [37, 38]. However, expression levels of p53BP1 or BRCA1 were not affected in HTLV-I-transformed MT-2 or C91PL before or after PJ-34 treatment (Fig. 8a, b). Increased expression of miR-107 was associated with PARPi sensitivity [39]. However, analyses of mature miR-107 expression revealed a significant increased expression in both resistant and sensitive HTLV-I-transformed cells (except for ED) when compared with HTLV-I-negative Jurkat T cells 


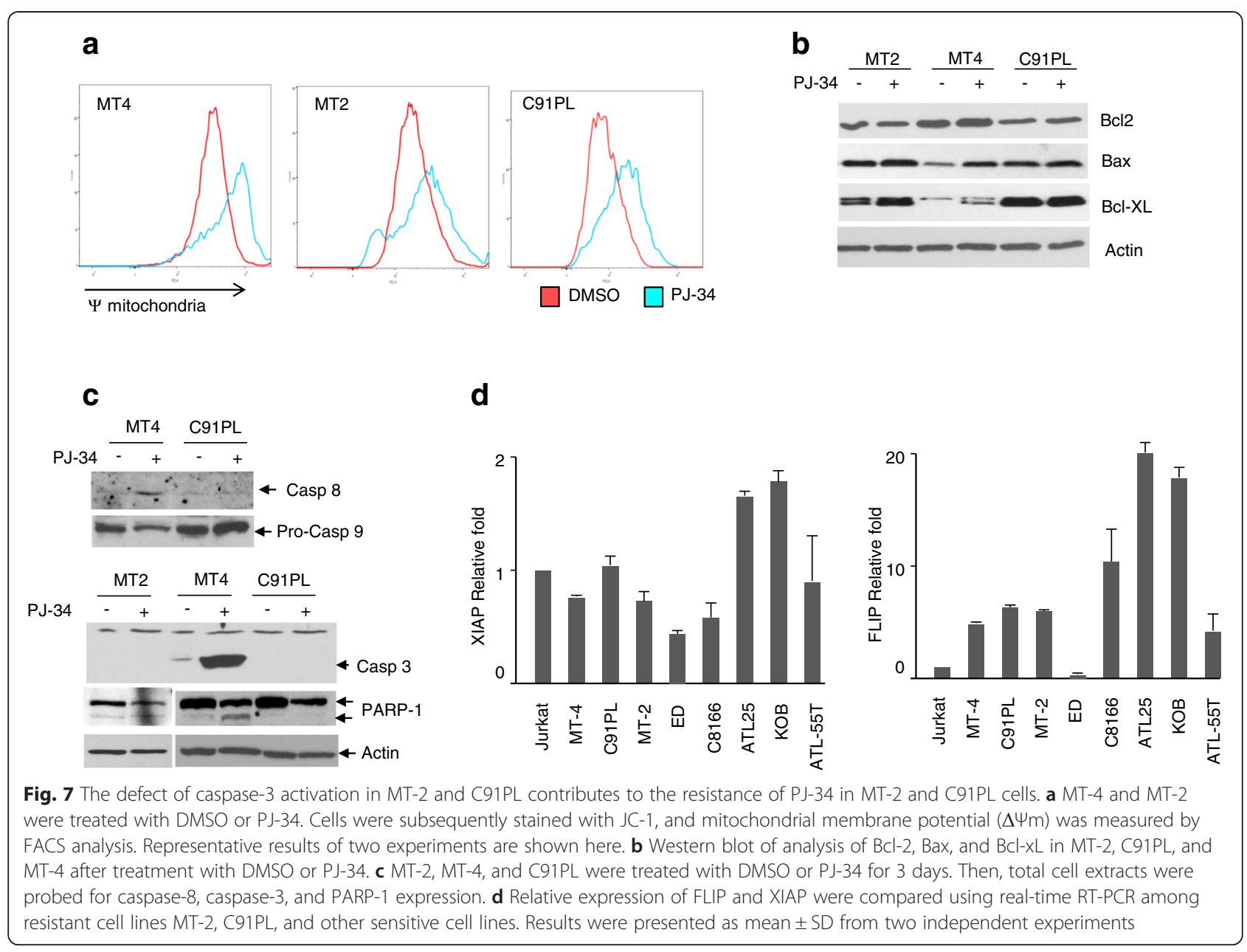

(data not shown). Similarly, the expression of PARP1 was not affected in MT-2-treated cells (Fig. 8c). In contrast, a significant increase in RelA/p65 was detected specifically in MT-2 but not MT-4 or C8166 cells following treatment with PJ-34, suggesting that canonical NF$\kappa \mathrm{B}$ activation may play an important role in resistance to PARPi in these cells.

\section{Discussion}

ATLL has a poor clinical outcome and current therapies have limited long-term benefits, in addition to most patients with acute or lymphoma ATLL having a short relapse-free survival. Patients diagnosed with chronic or smoldering forms of ATLL have a longer life expectancy but current treatments are ineffective and patients eventually progress to the acute type. Numerous studies have established that cancers with a dysfunction of the HR DNA repair pathway, also referred to as "BRCAness," share characteristics with BRCA1- or BRCA2-mutated cancer cells and are very sensitive to PARPi [40, 41]. We have previously reported that HTLV-I-transformed cells have pronounced defects in the homologous recombination (HR) DNA repair pathway. Additional studies demonstrated that tumor cells with a defective base excision repair (BER) pathway are prone to DDSB accumulation and hypersensitive to PARP targeting [42]. Notably, HTLV-I-transformed cells expressing the viral oncoprotein Tax have a defective BER pathway [43, 44]. These observations prompted us to investigate the potential benefits of PARPi therapy for ATLL.

In this study, we found a potent anti-proliferative effect of PARP small inhibitor PJ-34 against HTLV-Itransformed cells and patient-derived ATLL cells. Reduced tumor cell proliferation was demonstrated using XTT and CFSE staining assays. We showed that HTLVI-transformed cells arrested in the G2/M phase of the cell cycle following treatment with PJ-34 was consistent with alterations in cyclins and cyclin-dependent kinase inhibitor (CDKI) expression. The effect of PJ-34 was time- and dose- dependent and affected both IL-2dependent and IL-2-independent HTLV-I-transformed cells. We further demonstrated that PJ-34 is very effective against a panel of patient-derived ATLL cell lines, suggesting therapeutic potential. In general, anti- 


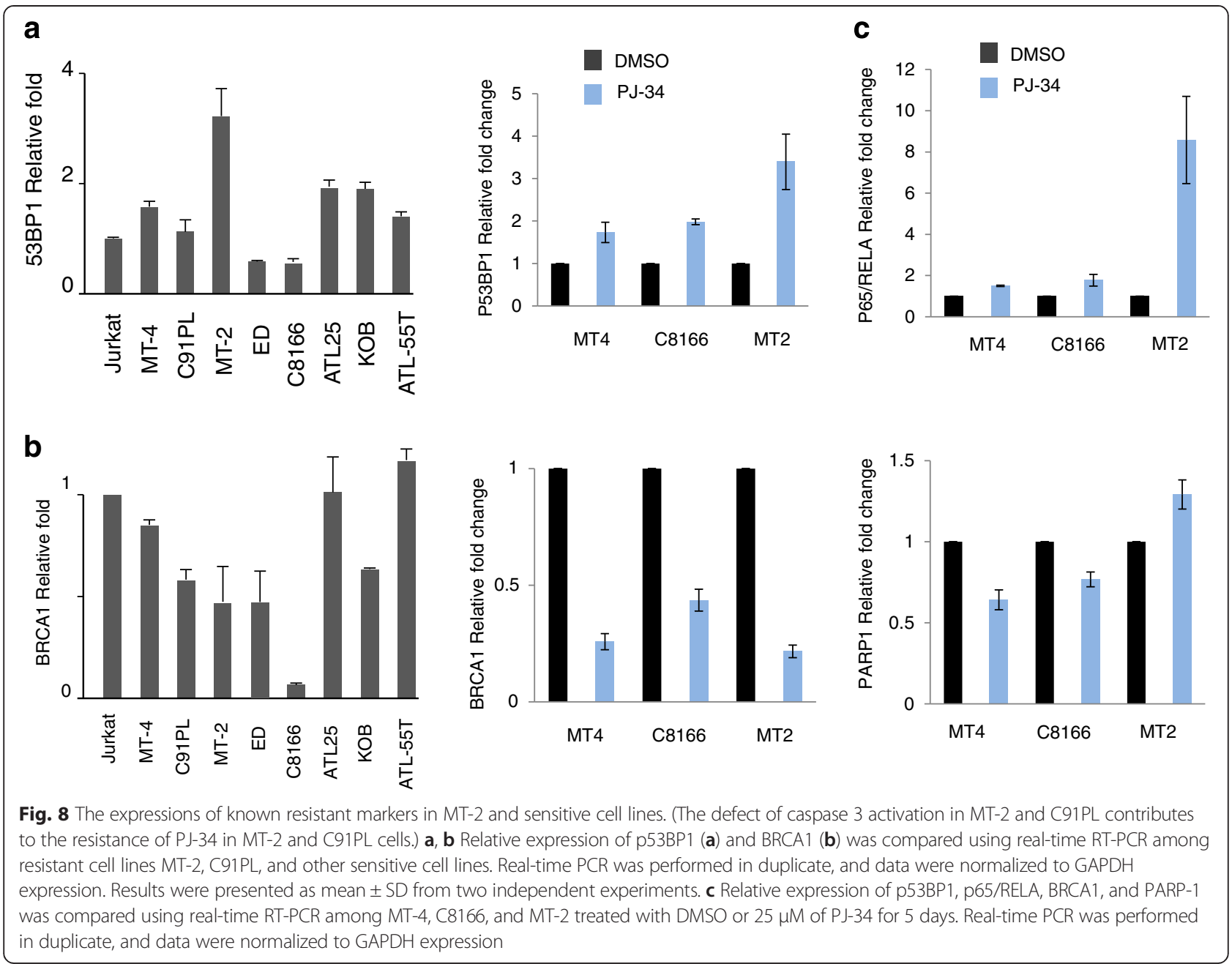

proliferative effects were associated with apoptotic cell death as shown by annexin V staining and the activation of caspase-3. We detected a loss of the mitochondrial membrane potential in HTLV-I cells following PJ-34 treatment, supporting the notion that PJ-34 induces cell death via the intrinsic mitochondrial pathway. Interestingly, treatment with PARPi PJ-34 resulted in accumulation of DNA breaks and reactivation of the tumor suppressor p53 transcriptional functions, as demonstrated by an increased expression of target genes p21WAF, MDM2, BAX, and GADD45. However, reactivation of p53 was not critical for PJ-34's effects since ATLL ED cells do not express p53 and were still sensitive to PJ-34. Interestingly, our studies identified two HTLV-I-transformed cell lines that were resistant to PJ34 treatment: MT-2 and C91PL. We then investigated differences between MT-4 (PJ-34 sensitive) and MT-2 and C91PL (PJ-34 resistant). We found no specific pattern of expression for p53BP1 or BRCA1 in PJ-34resistant versus sensitive cells. We then investigated expression of pro- and anti-apoptotic factors. Overall, anti- apoptotic proteins were not differentially affected but only MT-4 cells showed an increase in pro-apoptotic Bax expression. Bcl-xL, a pro-survival member of the Bcl-2 family, cannot directly inhibit Bax and specifically inhibits certain upstream BH3-only proteins. This supports our observation that the upregulation of Bcl-xL seen in MT-4 cells is unable to inhibit PJ-34-induced apoptosis and Bax activation, leading to apoptosis. We found that PJ-34-resistant cells were unable to activate caspase-3. This was unexpected because we and others previously demonstrated that MT-2 cells can activate caspase-3-dependent apoptosis [45-47].

Recently, PARP1 and PARP2 were found to play a role in sensing stalled or collapsed replication forks and to recruit the Mre11-Rad50-NBS1 (MRN) complex for resection and single-stranded DNA (ssDNA) formation, which allows RAD51 binding onto resected DNA to initiate HR $[48,49]$. Thus, PARP is also involved in HR repair at replication forks and inhibition of PARP leads to increased DNA lesions that can cause stalling and collapse of the DNA replication machinery. These observations 
suggest that targeting PARPi may be a good approach for ATLL patients. Combination therapy using PJ-34 and DNA-damaging agents such as cisplatin has shown promising results in other cancers and may be considered for a phase I trial of HTLV-I-associated ATLL. The reproducible growth arrest in cells from different genetic backgrounds highlights the potential value of PARPi as therapeutic agents for the treatment of ATLL.

\section{Competing interests}

The authors declare that they have no competing interests.

\section{Authors' contributions}

XTB and RM contributed equally to this work. XTB performed the experiments shown in Figures $1,5,6,7,8$, interpreted experiments, and wrote the manuscript. RM performed the experiments shown in Figures 2, 4, 7, 8, interpreted experiments, and wrote the manuscript. HCM performed the experiments shown in Figures 1, 3, 6, interpreted experiments, and wrote the manuscript. CN designed the project, interpreted the results and wrote the manuscript. All authors read and approved the manuscript.

\section{Acknowledgements}

The authors would like to thank Brandi Miller for editorial assistance.

\section{Financial support}

Research reported in this publication was supported by the National Cancer Institute of the National Institutes of Health under Award Number R01CA106258 to $\mathrm{CN}$. The content is solely the responsibility of the authors and does not necessarily represent the official views of the National Institutes of Health.

Received: 23 July 2015 Accepted: 13 October 2015

Published online: 23 October 2015

\section{References}

1. Poiesz BJ, Ruscetti FW, Gazdar AF, Bunn PA, Minna JD, Gallo RC. Detection and isolation of type $C$ retrovirus particles from fresh and cultured lymphocytes of a patient with cutaneous T-cell lymphoma. Proc Natl Acad Sci U S A. 1980;77:7415-9.

2. Tsukasaki K, Hermine O, Bazarbachi A, Ratner L, Ramos JC, Harrington Jr W, et al. Definition, prognostic factors, treatment, and response criteria of adult T-cell leukemia-lymphoma: a proposal from an international consensus meeting. J Clin Oncol. 2009;27:453-9.

3. Yasunaga J, Matsuoka M. Molecular mechanisms of HTLV-1 infection and pathogenesis. Int J Hematol. 2011;94:435-42.

4. Tsukasaki K, Tobinai K. Biology and treatment of HTLV-1 associated T-cell lymphomas. Best Pract Res Clin Haematol. 2013;26:3-14.

5. Ishitsuka K, Tamura K. Human T-cell leukaemia virus type I and adult T-cell leukaemia-lymphoma. Lancet Oncol. 2014;15:e517-26.

6. Sinha-Datta U, Horikawa I, Michishita E, Datta A, Sigler-Nicot JC, Brown M, et al. Transcriptional activation of hTERT through the NF-kappaB pathway in HTLV-I-transformed cells. Blood. 2004;104:2523-31.

7. Azimi N, Jacobson S, Leist T, Waldmann TA. Involvement of IL-15 in the pathogenesis of human T lymphotropic virus type l-associated myelopathy/ tropical spastic paraparesis: implications for therapy with a monoclonal antibody directed to the IL-2/15R beta receptor. J Immunol. 1999;163:4064-72.

8. Nicot C. Current views in HTLV-I-associated adult T-cell leukemia/lymphoma. Am J Hematol. 2005;78:232-9.

9. Gatza ML, Watt JC, Marriott SJ. Cellular transformation by the HTLV-I Tax protein, a jack-of-all-trades. Oncogene. 2003;22:5141-9.

10. Cheng H, Ren T, Sun SC. New insight into the oncogenic mechanism of the retroviral oncoprotein Tax. Protein Cell. 2012;3:581-9.

11. Pancewicz J, Taylor JM, Datta A, Baydoun HH, Waldmann TA, Hermine O, et al. Notch signaling contributes to proliferation and tumor formation of human T-cell leukemia virus type 1-associated adult T-cell leukemia. Proc Natl Acad Sci U S A. 2010;107:16619-24.

12. Migone TS, Lin JX, Cereseto A, Mulloy JC, O'Shea JJ, Franchini G, et al. Constitutively activated Jak-STAT pathway in T cells transformed with HTLV-I. Science. 1995;269:79-81.
13. Chaib-Mezrag H, Lemacon D, Fontaine H, Bellon M, Bai XT, Drac M, et al. Tax impairs DNA replication forks and increases DNA breaks in specific oncogenic genome regions. Mol Cancer. 2014;13:205.

14. Baydoun HH, Bai XT, Shelton S, Nicot C. HTLV-I tax increases genetic instability by inducing DNA double strand breaks during DNA replication and switching repair to NHEJ. PLoSOne. 2012;7:e42226.

15. Bryant HE, Schultz N, Thomas HD, Parker KM, Flower D, Lopez E, et al. Specific killing of BRCA2-deficient tumours with inhibitors of poly (ADPribose) polymerase. Nature. 2005;434:913-7.

16. Benafif $\mathrm{S}, \mathrm{Hall}$ M. An update on PARP inhibitors for the treatment of cancer. Onco Targets Ther. 2015;8:519-28.

17. O'Sullivan CC, Moon DH, Kohn EC, Lee JM. Beyond breast and ovarian cancers: PARP inhibitors for BRCA mutation-associated and BRCA-like solid tumors. Front Oncol. 2014;4:42.

18. Jasek E, Gajda M, Lis GJ, Jasinska M, Litwin JA. Combinatorial effects of PARP inhibitor PJ34 and histone deacetylase inhibitor vorinostat on leukemia cell lines. Anticancer Res. 2014;34:1849-56.

19. Gaymes TJ, Shall S, MacPherson LJ, Twine NA, Lea NC, Farzaneh F, et al. Inhibitors of poly ADP-ribose polymerase (PARP) induce apoptosis of myeloid leukemic cells: potential for therapy of myeloid leukemia and myelodysplastic syndromes. Haematologica. 2009;94:638-46.

20. Baydoun HH, Bai XT, Shelton S, Nicot C. HTLV-I tax increases genetic instability by inducing DNA double strand breaks during DNA replication and switching repair to NHEJ. PLoSOne. 2012;7:e42226.

21. Bellon M, Baydoun HH, Yao Y, Nicot C. HTLV-I Tax-dependent and -independent events associated with immortalization of human primary $T$ lymphocytes. Blood. 2010;115:2441-8.

22. Darzynkiewicz Z, Zhao H, Zhang S, Lee MY, Lee EY, Zhang Z. Initiation and termination of DNA replication during $S$ phase in relation to cyclins D1, E and A, p21WAF1, Cdt1 and the p12 subunit of DNA polymerase delta revealed in individual cells by cytometry. Oncotarget. 2015;6:11735-50.

23. Fukasawa K. p53, cyclin-dependent kinase and abnormal amplification of centrosomes. Biochim Biophys Acta. 2008;1786:15-23.

24. Lee MH, Yang HY. Regulators of G1 cyclin-dependent kinases and cancers. Cancer Metastasis Rev. 2003;22:435-49.

25. Miyazaki T, Arai S. Two distinct controls of mitotic cdk1/cyclin B1 activity requisite for cell growth prior to cell division. Cell Cycle. 2007;6:1419-25.

26. Vazquez-Ortiz G, Chisholm C, Xu X, Lahusen TJ, Li C, Sakamuru S, et al. Drug repurposing screen identifies lestaurtinib amplifies the ability of the poly (ADP-ribose) polymerase 1 inhibitor AG14361 to kill breast cancer associated gene-1 mutant and wild type breast cancer cells. Breast Cancer Res. 2014;16:R67.

27. Clark CC, Weitzel JN, O'Connor TR. Enhancement of synthetic lethality via combinations of ABT-888, a PARP inhibitor, and carboplatin in vitro and in vivo using BRCA1 and BRCA2 isogenic models. Mol Cancer Ther. 2012;11:1948-58.

28. Konstantinopoulos PA, Wilson AJ, Saskowski J, Wass E, Khabele D. Suberoylanilide hydroxamic acid (SAHA) enhances olaparib activity by targeting homologous recombination DNA repair in ovarian cancer. Gynecol Oncol. 2014;133:599-606.

29. Gartenhaus RB, Wang P. Functional inactivation of wild-type p53 protein correlates with loss of IL-2 dependence in HTLV-I transformed human T lymphocytes. Leukemia. 1995;9:2082-6.

30. Sakashita A, Hattori T, Miller CW, Suzushima H, Asou N, Takatsuki K, et al. Mutations of the p53 gene in adult T-cell leukemia. Blood. 1992;79:477-80.

31. Reid RL, Lindholm PF, Mireskandari A, Dittmer J, Brady JN. Stabilization of wild-type 553 in human T-lymphocytes transformed by HTLV-I. Oncogene. 1993;8:3029-36.

32. Datta A, Bellon M, Sinha-Datta U, Bazarbachi A, Lepelletier Y, Canioni D, et al. Persistent inhibition of telomerase reprograms adult T-cell leukemia to p53-dependent senescence. Blood. 2006;108:1021-9.

33. Su Z, Yang Z, Xu Y, Chen Y, Yu Q. Apoptosis, autophagy, necroptosis, and cancer metastasis. Mol Cancer. 2015;14:48.

34. Nicot C, Mahieux R, Takemoto S, Franchini G. BCl-X (L) is up-regulated by HTLV-I and HTLV-II in vitro and in ex vivo ATLL samples. Blood. 2000;96:275-81.

35. Kim H, Rafiuddin-Shah M, Tu HC, Jeffers JR, Zambetti GP, Hsieh JJ, et al. Hierarchical regulation of mitochondrion-dependent apoptosis by BCl-2 subfamilies. Nat Cell Biol. 2006;8:1348-58.

36. Javle M, Curtin NJ. The potential for poly (ADP-ribose) polymerase inhibitors in cancer therapy. Ther Adv Med Oncol. 2011;3:257-67. 
37. Jaspers JE, Kersbergen A, Boon U, Sol W, Van DL, Zander SA, et al. Loss of 53BP1 causes PARP inhibitor resistance in BRCA1-mutated mouse mammary tumors. Cancer Discov. 2013;3:68-81.

38. Oplustilova L, Wolanin K, Mistrik M, Korinkova G, Simkova D, Bouchal J, et al. Evaluation of candidate biomarkers to predict cancer cell sensitivity or resistance to PARP-1 inhibitor treatment. Cell Cycle. 2012;11:3837-50

39. Neijenhuis S, Bajrami I, Miller R, Lord CJ, Ashworth A. Identification of miRNA modulators to PARP inhibitor response. DNA Repair (Amst). 2013;12:394-402.

40. O'Sullivan CC, Moon DH, Kohn EC, Lee JM. Beyond breast and ovarian cancers: PARP inhibitors for BRCA mutation-associated and BRCA-like solid tumors. Front Oncol. 2014;4:42.

41. Lee JM, Ledermann JA, Kohn EC. PARP Inhibitors for BRCA1/2 mutationassociated and BRCA-like malignancies. Ann Oncol. 2014;25:32-40,

42. Horton JK, Stefanick DF, Prasad R, Gassman NR, Kedar PS, Wilson SH. Base excision repair defects invoke hypersensitivity to PARP inhibition. Mol Cancer Res. 2014;12:1128-39.

43. Philpott SM, Buehring GC. Defective DNA repair in cells with human T-cell leukemia/bovine leukemia viruses: role of tax gene. J Natl Cancer Inst. 1999;91:933-42

44. Kao SY, Marriott SJ. Disruption of nucleotide excision repair by the human T-cell leukemia virus type 1 Tax protein. J Virol. 1999;73:4299-304.

45. Sinha-Datta U, Taylor JM, Brown M, Nicot C. Celecoxib disrupts the canonical apoptotic network in HTLV-I cells through activation of Bax and inhibition of PKB/Akt. Apoptosis. 2008;13:33-40.

46. Suzuki Y, Ito S, Sasaki R, Asahi M, Ishida Y. Resveratrol suppresses cell proliferation via inhibition of STAT3 phosphorylation and Mcl-1 and CIAP-2 expression in HTLV-1-infected T cells. Leuk Res. 2013;37:1674-9.

47. Miyano-Kurosaki N, Ikegami K, Kurosaki K, Endo T, Aoyagi H, Hanami M, et al. Anticancer effects of phenoxazine derivatives revealed by inhibition of cell growth and viability, disregulation of cell cycle, and apoptosis induction in HTLV-1-positive leukemia cells. J Pharmacol Sci. 2009;110:87-97.

48. Ying S, Hamdy FC, Helleday T. Mre11-dependent degradation of stalled DNA replication forks is prevented by BRCA2 and PARP1. Cancer Res. 2012;72:2814-21.

49. Haince JF, McDonald D, Rodrigue A, Dery U, Masson JY, Hendzel MJ, et al. PARP1-dependent kinetics of recruitment of MRE11 and NBS1 proteins to multiple DNA damage sites. J Biol Chem. 2008;283:1197-208.

\section{Submit your next manuscript to BioMed Central and take full advantage of:}

- Convenient online submission

- Thorough peer review

- No space constraints or color figure charges

- Immediate publication on acceptance

- Inclusion in PubMed, CAS, Scopus and Google Scholar

- Research which is freely available for redistribution 